\title{
Inflammatory Bowel Disease at the Intersection of Autophagy and Immunity: Insights from Human Genetics
}

\author{
Natalia Nedelsky, Petric Kuballa, Adam B. Castoreno, and Ramnik J. Xavier
}

\begin{abstract}
Studies using human genetics have identified more than 160 loci that affect the risk of developing inflammatory bowel disease (IBD), including Crohn's disease (CD) and ulcerative colitis (UC). Several of these genes have been found to play key roles in the process of autophagy, a lysosome-based degradation pathway. Although historically considered to be a relatively nonselective process of degradation of cytosolic contents, autophagy has recently been revealed to have several selective and immune-specific functions that are relevant to the maintenance of intestinal homeostasis, including xenophagy, mitophagy, antigen presentation, secretion, and inflammasome regulation. In this chapter, we review the evidence that links autophagy-related genes, their immune-specific functions, and possible mechanisms of IBD pathogenesis. We summarize the basic molecular events underlying general and selective autophagy, and present evidence suggesting possible pathogenic mechanisms revealed by studies of IBD-associated risk alleles of ATG16L1 and IRGM. Finally, we review chemical biology-based experimental approaches for identifying autophagy regulatory pathways that may have implications for the development of therapeutics.
\end{abstract}

\footnotetext{
N. Nedelsky

Gastrointestinal Unit, Center for the Study of Inflammatory Bowel Disease, and Center for Computational and Integrative Biology, Massachusetts General Hospital, Boston, MA, USA

P. Kuballa · A. B. Castoreno · R. J. Xavier $(\bowtie)$

Gastrointestinal Unit, Center for the Study of Inflammatory Bowel Disease, and Center for Computational and Integrative Biology, Massachusetts General Hospital, Boston, MA, USA
}

Broad Institute of Harvard University and Massachusetts Institute of Technology,

Cambridge, MA, USA

e-mail: xavier@molbio.mgh.harvard.edu 


\section{Introduction}

Genome-wide association studies (GWAS) of IBD have been a major success story for genome-level studies of complex human disease. One of the particularly exciting results from these studies was the identification of autophagy as an unanticipated player in determining risk of IBD. This pathway, long considered to be a relatively nonselective process of bulk cytoplasmic degradation by the lysosome, is thought to have evolutionary roots as a mechanism to maintain metabolic homeostasis in response to decreased nutrient levels [1]. However, studies over the last 10 years have expanded the functions of autophagy to include roles in organelle clearance, antigen presentation, cell death, development, and the degradation of intracellular pathogens, among others. The role of autophagy in immunerelated processes [2-4] was particularly unexpected and has served as a guide in many studies seeking to connect human genetics to mechanisms of IBD pathogenesis [5-7].

These genetic studies have identified multiple genes within the autophagy network as playing a role in CD, including ATG16L1, IRGM, NOD2, and LRRK2 [8-11]. In the case of UC, the autophagy-related genes DAP1 and SMURF1 have also been identified as determinants of risk $[12,13]$. Further studies subsequently identified polymorphisms in $U L K 1, A T G 2 A$, and GABARAPL1 as harboring significant associations with $C D[14,15]$. Although autophagy has been implicated in the disease process in a range of other disorders, including neurodegenerative disease $[16,17]$, atherosclerosis, and cancer, to date no compelling genetic association has been found between autophagy and many immune-related diseases, including multiple sclerosis, celiac disease, rheumatoid arthritis, and psoriasis. However, targeted sequencing studies have also begun to identify coding mutations in essential autophagy genes as playing roles in disease, raising the possibility that more disease-associated autophagy-related polymorphisms have yet to be uncovered [18, 19].

In this chapter, we review studies that have employed human genetics to understand the link between autophagy and IBD. We begin by examining the idea of the intestinal mucosa as a physiological system wherein homeostasis is particularly vulnerable to disruptions in autophagy. We then present a basic overview of the molecular events involved in autophagy, followed by discussions of how autophagy may function as a selective process with critical roles in IBD-related immunity. These roles include (1) degradation of intracellular pathogens (xenophagy), (2) mitophagy, (3) antigen presentation, (4) secretion, and (5) inflammasome regulation. In the cases of xenophagy, mitophagy, and antigen presentation, we present detailed evidence of how autophagy functions in these pathways. Next, we review results from studies of two particularly well-examined CD risk single-nucleotide polymorphisms (SNPs), rs2241880 (ATG16L1, coding variant T300A) and rs13361189 (IRGM). Finally, we discuss approaches from the field of chemical biology that may be used to identify autophagy regulatory pathways with possible implications for therapeutics development. 


\section{Autophagy in the Intestinal Mucosa}

As mentioned above, autophagy has been implicated in several immune-related processes that influence IBD pathogenesis, including xenophagy, mitophagy, antigen presentation, secretion and vesicular trafficking, and cytokine-based regulation of inflammasome activity. One major point of intersection between these processes is their critical importance in the intestinal mucosa. This complex environment must maintain a delicate balance of immune responses, which are continuously stimulated by metabolic stresses and antigens from food. Microbes also play a major role in this milieu, which carries the highest bacterial load in the body. This tissue, therefore, may be especially susceptible to disruption in autophagy, with consequent alteration of homeostatic processes. For example, mice with conditional knockout of Atg7 in intestinal epithelial cells show increased severity of colitis when exposed to the infectious pathogen Citrobacter rodentium, while mice with deficiency in the core autophagy gene Atg1611 in hematopoietic cells show increased susceptibility to chemically induced models of colitis $[20,21]$.

Studies in human genetics have provided evidence that microbial stimulation may exist as a node of functional interaction between CD susceptibility genes, including ATG16L1 and NOD2. For example, expression of the ATG16L1 CD-associated risk allele (ATG16L1 T300A) results in impairment of autophagy and antigen presentation, both of which are enhanced by muramyl dipeptide (MDP), the bacterial ligand for the receptor NOD2 [22, 23]. Although no evidence has been found for an epistatic interaction between these two genes, this type of functional interaction suggests that autophagy may operate as a key point of intersection among multiple susceptibility genes.

\section{Basic Molecular Events in Autophagy}

The process of autophagy begins with an appearance of a flat membrane sheet termed the isolation membrane (also known as the phagophore assembly site in yeast), the origin of which remains unknown. The membrane elongates and expands to encompass its cargo, eventually fusing to form a double-membrane structure known as an autophagosome. The outer autophagosomal membrane then fuses with the lysosomal membrane, forming a degradative autolysosome. It should be noted that the source of the autophagosomal membrane is still a highly debated topic, and the existence of multiple sources cannot be excluded. In the case of the Endoplasmic reticulum (ER), a novel $\Omega$ (omega)-shaped vesicle called the omegasome has been identified, which resides on the ER and might precede the appearance of the isolation membrane [24].

Many of the molecular events underlying the assembly and elongation of autophagy membranes were initially identified by genetic screens in Saccharomyces cerevisiae $[25,26]$. These screens have identified approximately 35 core autophagy proteins, about half of which have clear homologs in mammals. For the purpose of 
this chapter, we divide these genes into five functional units: (1) the ULK complex (ATG1 complex in yeast), (2) ATG9, (3) Vps34 complexes, and (4-5) two ubiquitinlike conjugation systems anchored by ATG12 and ATG8, respectively.

The ULK complex (composed of ATG13, FIP200, and either ULK1 or ULK2) plays a central role in starvation-induced autophagy and is thought to be involved in the initiation of the autophagic cascade. The complex is recruited to sites of autophagosome formation, where it likely acts to recruit and disassemble other ATG protein complexes [27-30]. ATG9, a transmembrane protein, shuttles between autophagosomes, the trans-Golgi network, and late endosomes in a cycling process that may involve the ULK complex. ATG9 is of special interest to studies of xenophagy, since a recent report suggested that ATG9 is essential for the formation of the isolation membrane in anti-Salmonella autophagy in mouse embryonic fibroblasts (MEFs) [31]. In mammals, the Vps34 complex is likely required for the recruitment of several autophagy proteins to sites of autophagosome formation. This complex plays a critical role in supplying phosphatidylinositol 3-phosphate (PI3P) to the growing autophagosome, where it acts as an essential driving force in autophagosome assembly [32-36]. Furthermore, depending on the identity of its protein components, some alternative versions of the Vps34 complex may serve regulatory roles in autophagosome maturation and autophagosome-lysosome fusion [32-34, 37-39].

Two ubiquitin-like conjugation systems play important roles in autophagosome formation and maturation. The ATG12-based conjugation system is initiated by the E1-like ATG7, which activates and transfers ATG12 to the E2-like ATG10. ATG12 is then subsequently conjugated to ATG5 [40, 41]. ATG5 and ATG12 then bind with ATG16L1, forming a complex that is essential for autophagosome formation [42]. In addition to a key role in autophagosome formation, the second ubiquitin-like conjugation system may play additional roles in cargo recognition [43]. In this system, ATG8 is activated by the E1-like ATG7, is transferred to the E2-like ATG3, and is conjugated to phosphatidylethanolamine through the E3-like action of the ATG5ATG12 complex. The conjugation of phosphatidylethanolamine to ATG8 allows subsequent incorporation of ATG8 into the inner and outer membranes of autophagosomes [44-46]. Mammals appear to have two families of ATG8 homologs, known as LC3s and GABARAPs. While all ATG8 homologs can be incorporated into the autophagosomal membrane, the incorporation of LC3s and GABARAPs might occur at different stages, with LC3s acting at the stage of biogenesis and GABARAPs playing a more important role in autophagosome maturation [47]. Given that ATG8 remains associated with autophagosomes even after their fusion with lysosomes, tracking ATG8 and its homologs allows for localization of autophagosomes. Furthermore, since the inner membranes of autophagosomes are degraded upon formation of autolysosomes, tracking ATG8 enables the measurement of autophagic flux as reflected by ATG8 degradation [44, 45].

Several layers of regulation exist in the initiation and progression through the steps of autophagic degradation. One important regulatory influence is the cytoskeleton. Autophagosome trafficking involves movement along microtubules, which facilitates fusion with lysosomes [48, 49]. Changes in lysosome positioning regulate mTORC1 signaling, since peripheral localization of lysosomes and 
mTORC1 increases mTORC1 activity, while perinuclear lysosomal clustering decreases activity [49]. Heat shock protein beta 1 (HSPB1), a protein that interacts with and mediates reorganization of the actin cytoskeleton, is also linked to mitophagy [50]. Disruption of HSPB1 signaling can lead to accumulation of dysfunctional mitochondria, mitochondrial fragmentation, and effects in mitochondrial respiration in MEFs [51].

Transcription also serves as a regulatory influence in the autophagic process. Autophagosome and lysosome biogenesis appear to be transcriptionally coregulated by the transcription factor EB (TFEB) [52]. Overexpression of TFEB induces autophagy, while a block of TFEB expression by RNAi decreases autophagy. Interestingly, TFEB action is implicated in basal as well as starvation-induced autophagy and therefore TFEB might represent a transcriptional master regulator of the autophagy pathway. Furthermore, translocation of TFEB from the cytosol to the nucleus is strongly enhanced upon removal of nutrients, suggesting that TFEB function is sensitive to nutrients and growth factors. Under fed conditions, phosphorylation on serine 142 of TFEB can be mediated by the extracellular signal-regulated kinase 2 (ERK2) and might be sufficient to retain TFEB in the cytosol. A comprehensive analysis of 51 genes with known functions in the autophagy pathway shed some light on transcriptional regulation of autophagy in the context of starvation and TFEB function. Strikingly, TFEB-dependent transcription patterns generally correlate with starvation effects, further emphasizing the role of TFEB as an autophagy regulator that can directly or indirectly sense nutritional status [52]. However, the transcription of the majority of genes is not significantly affected by starvation or TFEB expression levels [52], suggesting that transcription factors other than TFEB control the expression of most autophagy and autophagy-related genes, possibly in a nutrient-insensitive fashion.

\section{Xenophagy}

Autophagy has now been well described as playing a key role in the degradation of intracellular pathogens, including bacteria, in a specialized process known as xenophagy. However, the environment of the intestinal mucosa provides special challenges to this function. As this tissue is exposed to both commensal and pathogenic bacteria, the interactions between these flora and host immune responses must be tightly controlled.

\section{Molecular Basis of Xenophagy}

The molecular events surrounding the process of xenophagy follow the same basic pattern as those involved in autophagic degradation of other cellular contents, such as organelles or aggregated protein deposits. However, the mechanisms underlying 
the selection of cargo for degradation by xenophagy remain largely unknown. Components of the innate immune response can interact with autophagy proteins to target bacteria for autophagic degradation. Mechanisms involved in xenophagy of other species of bacteria may share some components with anti-Salmonella xenophagy, but to date, detailed molecular mechanisms have been best explored for Salmonella.

Upon infection of epithelial cells, S. typhimurium resides in Salmonellacontaining vacuoles (SCVs). The environment of the SCV promotes bacterial survival and replication. The SCV protective niche is established by effectors of two type III secretion systems (T3SSs) encoded by Salmonella pathogenicity islands 1 and 2 (SP-1 and SP-2). The secretion and activity of T3SS components is highly regulated both temporally and spatially. For example, $\mathrm{pH}$ sensing regulates the transition from translocon protein secretion and pore formation to effector secretion [53]. Once secreted into the host cell, the activity of effectors can be modulated. For example, the T3SS SP-1 effectors SopA, SopE, SptP, and SopB are all ubiquitinated shortly after infection by the host cell machinery [54]. The phosphoinositide phosphatase activity of SopB regulates several processes that promote internalization and survival of $S$. typhimurium, including cytoskeletal rearrangements that promote invasion, SCV biogenesis and maturation, and prosurvival Akt activation [54]. The ability of SopB to regulate these functions is dependent upon its ubiquitination status $[54,55]$. A SopB mutant that cannot be ubiquitinated has a number of defects, including a failure to relocalize from the plasma membrane to $\mathrm{SCV}$, disrupted recruitment of Rab5 to SCVs, and perturbed S. typhimurium replication [54]. Thus, ubiquitination-dependent relocalization of SopB to SCVs promotes the establishment of SCVs as a niche environment for replication. This is due in part to the ability of SopB to reduce the net negative charge of PI(4,5)P2 and phosphatidylserine lipids on SCV membranes, thereby disrupting maturation and fusion with lysosomes [55].

Although most $S$. typhimurium resides in SCVs, a small but significant fraction is released into the cytosol from damaged SCVs. This cytosolic S. typhimurium rapidly becomes ubiquitinated. Ubiquitinated bacteria may be recognized by selective cargo receptors, including p62 and NBR1, which act as bridging factors that bind to both ubiquitin and the autophagosome. p62 and NBR1 share a similar protein structure and domain composition and may show some level of redundancy, particularly in degradation of protein aggregates (aggrephagy) and mitochondria (mitophagy). However, while p62 is important for the autophagic clearance of Salmonella, NBR1 is not [56]. Therefore, the mechanism of xenophagy, which relies on ubiquitination events on or around intracellular bacteria, might differ from other ubiquitin-dependent selective autophagy events. Interestingly, nuclear dot protein 52 (NDP52), in addition to p62, has been implicated in the recognition of ubiquitin-coated invasive bacteria, but not in other forms of selective autophagy [57]. NDP52 contains a putative LIR motif at its N-terminus, can directly bind ubiquitin through a C-terminal ubiquitin-binding zinc finger, and can form homooligomers via its coiled-coil domain [57-59]. p62 and NDP52 appear to act cooperatively in the recognition and killing of Salmonella enterica serotype Typhimurium 
(S. typhimurium), Listeria monocytogenes, and Shigella flexneri [60, 61]. However, p62 and NDP52 are recruited to distinct microdomains of invading Salmonella, suggesting that ubiquitin coats on Salmonella might be heterogeneous [60].

Interestingly, in macrophages but not in epithelial cells, proteasomes localize to ubiquitin-coated Salmonella with potential effects on efficient rupture of damaged SCVs and major histocompatibility complex (MHC) class I antigen presentation $[62,63]$. Taken together, ubiquitin-dependent autophagic targeting of intracellular pathogens and by-products has implications for pathogen growth and the regulation of inflammatory responses. However, in most cases, the E3 ubiquitin ligases, the nature of ubiquitin modifications, and the substrates involved in ubiquitin-dependent xenophagy are not known and may be pathogen- as well as cell type-specific. One important exception is LRSAM1, an LRR- and RING-domain protein that was recently identified as an E3 ubiquitin ligase crucial for ubiquitin-dependent autophagy of S. typhimurium [64]. Interestingly, LRSAM1 was required for ubiquitination of intracellular bacteria but dispensable for ubiquitination of aggregated proteins, confirming that LRSAM1 serves as a selective and novel antibacterial sensor that mediates target selection for the xenophagy pathway. To date, however, LRSAM1 remains the only known ubiquitin ligase associated with antibacterial autophagy, and a detailed biochemical characterization of the aforementioned factors will yield critical insights into host-pathogen interactions and may boost the development of novel pharmaceuticals aimed to combat infectious diseases.

\section{Xenophagy and IBD Risk Genes}

As mentioned above, intestinal tissues are in constant interaction with both commensal and pathogenic bacteria. To prevent inappropriate responses to commensal bacteria, the cells of the intestinal mucosa must be educated to tolerate these microbes and their products [65]. Breakdown of this tolerance, with consequent inappropriate inflammation, has been proposed to play a major role in the pathogenesis of IBD. This model is supported by the finding that the IBD-associated genes, $A T G 16 L 1, I R G M$, and $U L K 1$, all play roles in the process of xenophagy. ATG16L1, $I R G M$, and $U L K 1$ each function in the degradation of $S$. typhimurium, while ATG16L1 and IRGM also play a role in antibacterial autophagy of a CD-relevant strain of Escherichia coli (adherent invasive E. coli, or AIEC) [10, 66-69]. ULK1 may also function in the degradation of L. monocytogenes. Furthermore, the function of LRRK2 in reactive oxygen species (ROS)-driven bacterial killing, as well as its role in autophagy, raises the possibility that this protein may also be involved in xenophagy of gut bacteria [70-72]. Defects in these gene products might lead to impaired bacterial handling, promoting an environment of chronic inflammation and inappropriate host responses to commensal bacteria.

Some intracellular pathogens have also been highlighted as potential contributors to risk of $\mathrm{CD}$, including mycobacteria. Genetic loci that include IRGM, $L R R K 2$, and NOD2 have been associated with increased susceptibility to leprosy 
and tuberculosis, suggesting an interesting point of interaction between autophagy genes, CD, and mycobacteria. These studies, combined with reports that autophagy may play a key role in control of Mycobacteria tuberculosis, have led to the suggestion that control of mycobacteria might be altered in CD [73, 74]. Genetic studies appear to support this idea, especially in the context of granulomas, a histological hallmark of both $\mathrm{CD}$ and tuberculosis: polymorphisms in loci containing the autophagy genes ATG2A and GABARAPL1 have been linked to granulomas in $\mathrm{CD}$ patients. To date, however, connections between $\mathrm{CD}$ and the mycobacterioses remain largely speculative, to be answered perhaps by future well-powered GWAS for tuberculosis [75].

The recent wealth of data from the human microbiome project has also highlighted how little we understand the complex relationship between IBD and microbial stimulation. Certainly the diversity and composition of the gut microbiota are major factors influencing gut homeostasis, and particular dietary nutrients and metabolites are likely to interact with host genetics to influence host-microbiome interactions. An imbalance in the composition of the gut microbiome, termed dysbiosis, has been associated with IBD, including shifts in relative abundances of bacterial taxa, decreases in the diversity of the bacterial community, and alterations in the functional composition of the microbiome.

\section{Mitophagy}

The autophagic processing of mitochondria (mitophagy) was directly implicated in the context of IBD by a recent study that identified SMURFI as a susceptibility gene for UC [13]. This gene is an ubiquitin ligase that was recently identified, through an image-based genome-wide siRNA screen, as an important mediator of selective viral autophagy and mitophagy [76].

\section{Molecular Basis of Mitophagy}

In contrast to other substrates for selective autophagy, for example surplus peroxisomes or ribosomes, mitophagy is well studied and underlying biochemical mechanisms for this process are emerging. Upon loss of mitochondrial membrane potential, PTEN-induced putative kinase 1 (PINK1) accumulates on the outer mitochondrial membrane $[77,78]$. This step is accompanied by stabilization of PINK1 protein and may involve a membrane potential-dependent block of PINK1 proteolysis by the mitochondrial inner membrane protease presenilin-associated rhomboidlike protein (PARL). This protease is effective in healthy mitochondria but impaired in damaged mitochondria [77, 79]. PINK1 then recruits Parkin1 to damaged mitochondria, which results in Parkin1-dependent ubiquitination of some mitochondrial proteins such as voltage-dependent anion channel 1 (VDAC1) and mitofusins [80-83]. p62 accumulates around ubiquitin-decorated damaged mitochondria and 
PB1-domain-mediated oligomerization of p62 results in mitochondrial clustering, eventually leading to autophagic degradation of mitochondria [80]. It should be noted that the mitochondrial ubiquitination event required for mitophagy may involve multiple substrates and/or may be cell type-specific, as p62 recruitment to damaged mitochondria and mitophagy is not impaired in VDAC1-deficient MEFs [78]. In addition, activating molecule in Beclin 1-regulated autophagy (Ambra1), an autophagy-regulating protein initially identified in neuronal cells [84], is recruited to damaged mitochondria in a Parkin1-dependent fashion [85] and knockdown of Ambra1 expression results in impairment of mitophagy. Interestingly, Ambra1 recruitment most likely involves direct binding to Parkin1, but Ambra1 is not a substrate for Parkin1-mediated ubiquitination [85]. Thus, Parkin1-mediated mitophagy relies at least in part on effects other than ubiquitination.

\section{Mitophagy and IBD Risk Genes}

As mentioned above, the best evidence linking mitophagy to IBD comes from the identification of SMURF1 as a gene associated with susceptibility to UC. SMURF1 is a HECT domain-containing E3 ubiquitin ligase that interacts with the selective autophagy factor p62 [76]. Smurf1 $1^{-1}$ MEFs show defects in the degradation of Sindbis and herpes simplex virus as well as impaired clearance of damaged mitochondria. Supporting this observation, Smurfl-deficient mice display an accumulation of damaged mitochondria in the heart, brain, and liver. Interestingly, Smurf $1^{-1-}$ MEFs are competent for starvation-induced bulk autophagy, indicating that SMURF1 is a specific mediator of selective autophagy. However, a mechanistic link between SMURF1 polymorphisms and IBD pathogenesis remains to be examined.

\section{Antigen Presentation}

Evidence for a link between IBD, antigen presentation, and autophagy originates from studies of CD-associated risk variants in NOD2 and ATG16L1. Although a role for autophagy in antigen presentation has been well described in basic studies, how this function may be altered in the context of IBD remains relatively unknown.

\section{Molecular Basis of Autophagy in Antigen Presentation}

Major histocompatibility complex (MHC) molecules on the cell surface present peptide antigens to $\mathrm{T}$ cells. MHC class I molecules interact with the $\mathrm{T}$ cell receptor (TCR) of $\mathrm{CD}^{+}$cytotoxic T cells, while MHC class II molecules interact with the TCR of $\mathrm{CD}^{+}$helper T cells. MHC class I antigens are derived from proteasomal 
degradation of cytosolic proteins. In contrast, MHC class II antigens are delivered to the lysosome before transfer to the MHC class II loading compartment and transport to the cell surface. Delivery of these antigens to the lysosome can occur either via endocytosis of protein antigens from the extracellular space or by autophagy of cytoplasmic material [86-88]. Autophagy also plays an important role in the presentation of pathogen antigens by dendritic cells (DCs). Mice lacking Atg5 specifically in DCs show impaired $\mathrm{CD}^{+}{ }^{+} \mathrm{T}$ cell priming following infection with herpes simplex virus, an impairment that is due to a decreased ability of Atg5-deficient DCs to process and present antigens for presentation on MHC class II molecules [89]. Furthermore, these cells show delayed fusion of phagosomes to lysosomes. Autophagic degradation may also combine with vesicular trafficking to facilitate presentation of citrullinated self-peptides by DCs to $\mathrm{CD}^{+} \mathrm{T}$ cells. This finding is particularly relevant to IBD, since immune responses against citrullinated selfproteins are associated with autoimmunity [90, 91].

\section{Antigen Presentation and IBD Risk Genes}

Interestingly, DCs from patients with CD-associated risk variants of ATG16L1 or NOD2 show defects in autophagy induction, bacterial trafficking, and MHC class II antigen presentation to $\mathrm{CD}^{+} \mathrm{T}$ cells [23]. Furthermore, autophagy was recently reported to destabilize the immune synapse between DCs and T cells; in this report, decreased expression of ATG16L1 or IRGM resulted in hyperstable interactions between DCs and T cells as well as increased activation of T cells, suggesting a mechanism by which adaptive immunity might be increased in patients with $C D$ who carry ATG16L1 risk alleles [92].

\section{Vesicular Trafficking and Secretion}

The suggestion that the secretory pathway might be modulated by autophagy in the context of CD arose from the GWAS-based identification of ATG16L1 as a common $\mathrm{CD}$-associated risk gene. Patients carrying the $\mathrm{CD}$-associated variant of this gene (ATG16L1 T300A), as well as Atg 1611 hypomorphic (Atg1611 ${ }^{\mathrm{HM}}$ ) mice, were found to show abnormalities in specialized epithelial cells called Paneth cells. These cells play a central role in innate immunity and are an important source of antimicrobial peptides in the small intestine, serving to prevent microbial invasion and control the composition of the gut microflora [93]. ATG16L1 T300A patients and $\operatorname{Atg} 16 l 1^{\mathrm{HM}}$ mice exhibit decreased numbers of granules and diffuse staining for lysozyme in their Paneth cells, raising the possibility that autophagy plays a key role in secretion in these cells [94, 95]. Supporting this hypothesis, mice lacking Atg5 or Atg7 in intestinal epithelial cells also show Paneth cell defects [96]. Interestingly, the Paneth cell phenotype in Atg $1611^{\mathrm{HM}}$ 
mice can be influenced by exposure to a pathogen, since the cellular defects can be eliminated by maintaining mice in a virus-free environment [94]. Paneth cell defects have also been observed in mice lacking other CD-associated genes, including Nod2 and Xbpl [97, 98]. More recently, a role was reported for Paneth cells in sensing nutrient availability, demonstrating that caloric restriction reduces Paneth cell-specific signaling by mTORC1, a regulator of autophagy [99]. Taken together, these reports suggest that Paneth cell homeostasis and function are intimately linked to autophagy. However, whether autophagy directly regulates vesicular trafficking and secretion remains unknown, since the molecular mechanisms by which ATG16L1 might regulate these cellular functions are poorly understood.

\section{Inflammasome Hyperactivation and Cytokine Secretion}

Inflammasomes are molecular scaffolds that activate caspase 1 and maturation of the proinflammatory cytokines interleukin (IL)-1 $\beta$ and IL-18. Several types of inflammasomes have been described, which are activated by a number of endogenous and exogenous signals.

Recent studies have shown that autophagy defects can lead to inflammasome hyperactivation. For example, stimulation of Atg $1611^{-/-}$fetal macrophages with lipopolysaccharide (LPS) induces elevated levels of activate caspase 1 and enhanced secretion of IL-1 $\beta$ and IL-18 [21]. Mice with specific deletion of Atg1611 in hematopoietic cells also show higher serum levels of IL-1 $\beta$ and IL-18 in response to dextran sulfate sodium [100], accompanied by increased inflammation and mortality.

The underlying mechanism linking autophagy to regulation of the inflammasome remains unknown. One possibility is that defects specifically in mitophagy result in increased levels of ROS, which are known to cause hyperactivation of

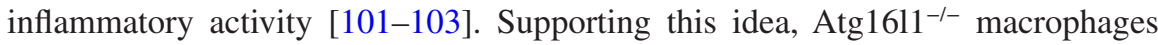
treated with a ROS scavenger did not show the increased IL- $1 \beta$ secretion described above [21].

Samples from patients have also shown ATG16L1 coding variant-dependent alterations in IL-1 $\beta$ secretion. In these studies, stimulation of peripheral blood mononuclear cells with MDP, but not LPS, resulted in a relative increase of IL-1 $\beta$ secretion from ATG16L1 T300A-expressing cells. Although no difference was found in levels of activated caspase 1 , protein levels of pro-IL- $1 \beta$ and mRNA levels of IL-1 $\beta$ were increased in the context of the ATG16L1 risk allele [104].

The autophagy adapter p62 may also provide a connection between IL-1 $\beta$ signaling and ATG16L1 [105]. In a recent study, we showed that levels of p62 are normally regulated by ATG16L1-based activation of the ubiquitin ligase Cullin-3, which promotes proteasomal degradation of p62. Loss of ATG16L1, however, can result in decreased levels of Cullin-3 and decreased degradation of p62. As p62 can act as a scaffold in the IL- $1 \beta$ signaling pathway, elevated levels of p62 may result in amplified IL-1 $\beta$ signaling [105]. 


\section{ATG16L1 T300A}

One of the best-studied CD-associated risk alleles is the T300A coding polymorphism in ATG16L1. Atg $1611^{-/-}$mice are not viable [21], but an alternative hypomorphic model (Atg1611 ${ }^{\mathrm{HM}}$ ) has yielded important clues to how the T300A coding polymorphism may result in altered autophagy and increased inflammation $[94,95]$. The site of the T300A polymorphism is near a WD-repeat domain, which is present in mammalian ATG16L1 but absent in yeast Atg16. This domain is not required for autophagy, consistent with the finding that the T300A polymorphism does not affect classical autophagy $[42,67]$.

As a core autophagy protein, $A T G 16 L 1$ has been shown to play multiple roles in IBD-relevant processes, including xenophagy, antigen presentation, IL-1 $\beta$ production, and secretion (see Fig. 1). Interestingly, the CD-associated risk allele appears
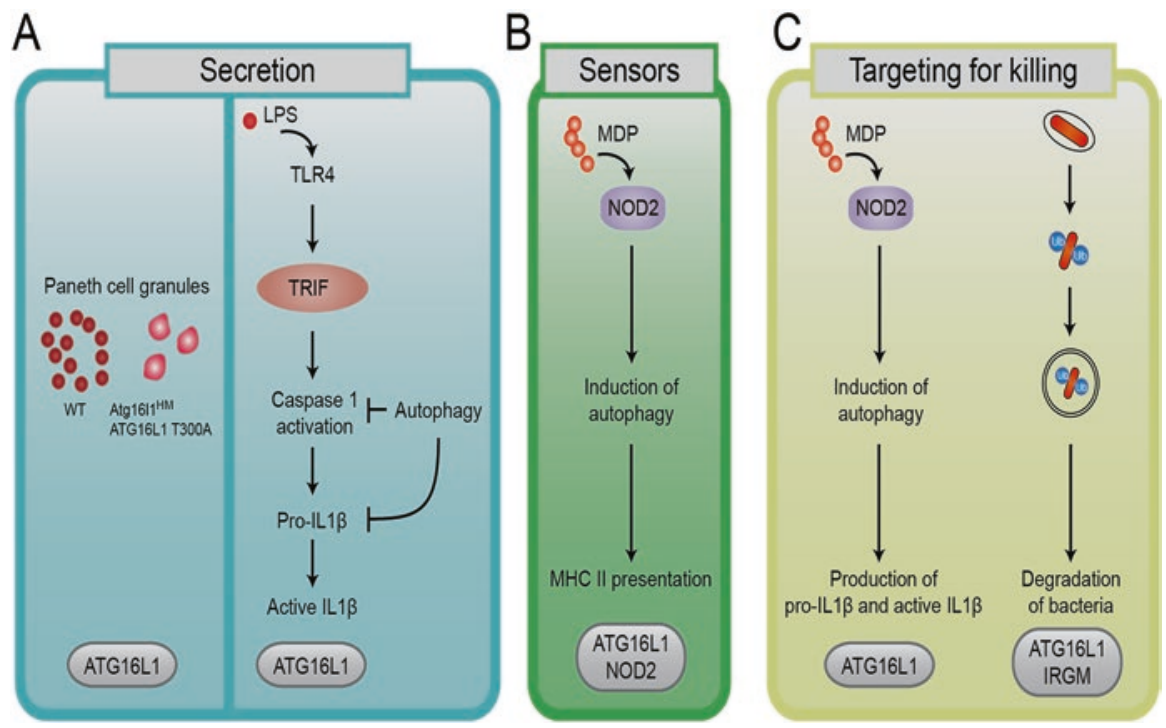

Fig. 1 Immune-related autophagy functions. (a) Paneth cells from CD patients expressing the CD-associated ATG16L1 T300A allele, as well as mice expressing a hypomorphic allele of Atg16l1 (Atg16l1 $\left.{ }^{H M}\right)$, show altered granule morphology, suggesting a role for autophagy in secretion of antimicrobial peptides. Another role for autophagy in secretion is suggested by observations in macrophages, in which secretion of active IL-1 $\beta$ (as well as active IL-18, not shown) upon LPS stimulation is regulated by autophagy at two levels. In this pathway, loss of ATG16L1 expression is associated with increased cytokine secretion. (b) Autophagy can also act as a sensor for bacterial products. In dendritic cells, activation of NOD2 by MDP induces autophagy that leads to MHC class II antigen presentation. This pathway appears to be altered in the context of CD-associated SNPs in ATG16L1 and NOD2. (c) Autophagy plays a role in targeting bacteria and bacterial products for degradation/killing. In one pathway, observed in primary blood mononuclear cells, activation of NOD2 by MDP induces autophagy that leads to production of pro-IL-1 $\beta$ and secretion of active IL- $1 \beta$. The CD-associated variant ATG16L1 T300A is associated with increased amounts of active IL- $1 \beta$ upon MDP stimulation. Autophagy can also directly degrade bacteria. In epithelial cells, bacteria become ubiquitinated and targeted for autophagic degradation in a process that requires the CD-associated genes ATG16LI and IRGM 
to have different effects on these functions depending on the cell type examined $[42,67]$. It is perhaps not surprising, then, that T300A has also been associated with defects in antigen presentation increases in IL-1 $\beta$ production, and abnormalities in Paneth cell secretion in patient samples [23, 95, 104]. In addition, antibacterial autophagy of Salmonella is affected by the presence of the ATG16L1 CD risk allele [67] and patients with the T300A allele show increased susceptibility to infection by Helicobacter pylori [106]. These findings illustrate that disease genes are likely to have specific functions in specific cell types, and researchers must consider the cell type- and stimulation-specific contexts used to examine diseaseassociated phenotypes.

\section{IRGM rs13361189}

IRGM (immunity-related GTPase family $\mathrm{M}$ ) is a human gene recently identified as playing an important role in antibacterial and antiviral autophagy. To date, xenophagy of pathogens including S. typhimurium, M. tuberculosis, and CD-associated AIEC has been reported to be dependent on IRGM $[66,68,69,107]$.

A synonymous SNP within the human IRGM locus (rs13361189) is associated with $\mathrm{CD}$, and a $20 \mathrm{~kb}$ deletion polymorphism within the $5^{\prime}$ untranslated region of $I R G M$ is in perfect linkage disequilibrium with this SNP [68, 108]. However, both rs13361189 and the deletion polymorphism have also been reported to be in perfect linkage disequilibrium with a synonymous exonic SNP (rs10065172). This SNP is associated with decreased binding of the microRNA miR-196 to the $3^{\prime}$ UTR of $I R G M$, an event that downregulates expression of $I R G M$ [69]. This finding is consistent with the observation that expression of miR-196 is increased in epithelial cells of the inflamed ileum and colon of CD patients compared with controls [69]. This result may be relevant to autophagic targeting of pathogenic bacteria, since knockdown of IRGM or overexpression of miR-196 affects the autophagic targeting of Adherent-invasive Escherichia coli (AIEC). Furthermore, an additional IRGM polymorphism (-261TT) is associated with an increase of IRGM expression and protection against M. tuberculosis [74].

\section{Autophagy Interaction Network}

Despite the approaches described in the studies above, in which individual autophagy genes are examined in relative isolation, it is important to note that a complex network of autophagy-related proteins exists in the cell and that cellular functions captured within this network likely extend beyond the strict bounds of lysosomal degradation.

As mentioned above, autophagy is implicated in many cellular pathways, including adaptations to changes in environmental factors such as nutrient 
availability. Many autophagy and autophagy-related proteins have been characterized, and distinct autophagic complexes with functions in autophagosome assembly and maturation have been identified and analyzed in great detail. The current picture suggests a stepwise process, but the precise regulation of autophagy in changing environmental settings - including fluctuations in growth factor supply, cytokine signaling, and the presence of pathogens - likely involves complex cross-talk between pathway components. Behrends and colleagues recently used an approach which might be described as "pathway proteomics" to investigate the network organization of the human autophagy system [109]. In doing so, they provided a basis for understanding the autophagy network on a protein level. In brief, interaction partners for 32 epitope-tagged stably expressed proteins, representing core autophagy as well as autophagy-related processes, were identified using mass spectrometry following immunoprecipitation of the respective tagged protein. A subset of proteins interacting with primary baits, were used as baits in secondary screens, leading to the identification of 751 high-confidence candidate interactions between a total of 65 baits and an additional 409 proteins. A high coverage of known interactions, a high level of validation (reciprocal immunoprecipitations and in vitro assays), and validation of autophagy functions of network components via RNAi allow for the designation of these results as a true autophagy protein interaction network. Interestingly, different autophagy subnetworks such as the ULK complex, Vps34 complex, ATG8 conjugation system, and PI3P signaling members were revealed to have an underappreciated high level of interconnectivity. Novel interaction partners for the mammalian ATG8 homologs were also identified. These interactors may be shared among all ATG8 homologs, specific for LC3 or GABARAP subfamily members, or specific for individual ATG8 homologs. However, differential cellular abundance of distinct ATG8 homologs could in part account for different efficiencies with respect to the coimmunoprecipitation of ATG8 interaction partners [109]. Nevertheless, differences in LC3-interacting region (LIR) motif composition among different ATG8 homologs can affect binding to p62, NBR1 [110], and possibly control differential requirements of LC3 and GABARAP subfamily members in early or late phases of autophagosome biogenesis [47]. In addition, it will be interesting to see if some of the novel ATG8 interactors might function as receptors for certain types of selective autophagy. Good candidates might be recovered by a comparison of ATG8 interactors identified by these proteomics studies and a list of proteins predicted to combine LIR and ubiquitin-binding motifs (Kay Hofmann, unpublished data in [111]).

Interestingly, pharmacological induction of autophagy led to alterations of protein-protein interactions within some, but not all subnetworks [109]. It will be interesting to see if such clusters might be targets of microRNAs, which generally modify the translation of multiple genes at the same time. In addition, it is possible that different subnetworks are altered by different stimuli, possibly in a celltype-specific fashion, which could ultimately help in the design of disease-specific autophagy-enhancing or -inhibiting drugs. 


\section{Chemical Modulators of Autophagy}

As highlighted throughout this chapter, many unanswered questions remain regarding the mechanistic basis of multiple autophagy functions. We believe that the field of chemical biology is poised to help answer these questions, providing the tools to dissect the regulatory nodes that exist at the intersections of autophagy and immunity. Furthermore, studies in this field may help to identify druggable targets within the autophagy pathway and its regulatory influences.

Small molecules have been proven to be useful tools in autophagy research, demonstrating success by increasing our knowledge of autophagy regulation while simultaneously identifying potential therapeutic compounds. Small molecule screens are particularly important in identifying probes for dissecting complex biological processes such as autophagy, as they allow the dissection of multiple steps in a pathway, provide temporal control over target function, and are often reversible. These screens are also particularly powerful in enabling researchers to identify molecules that parallel gene activity. Furthermore, by pursuing the mechanism of action (MOA) of active compounds, novel regulatory pathways can be identified.

Target identification and MOA determination is a rate-limiting step to small molecule discovery in phenotype-based screens and relies on the integration of multiple complementary approaches. One such approach is candidate-based, which can be used to determine whether novel small molecules target known MOAs. Several unbiased approaches to determine small molecule MOAs can also be used, including (i) combining quantitative proteomics (SILAC) with affinity enrichment to identify proteins that interact with the small molecule and yield the observed phenotype, (ii) compound profiling and connectivity analysis using gene expression signatures, and (iii) genetic complementation of small molecule effects by RNAi or overexpression screening to identify genes that function in the same pathway as the small molecule [112, 113].

Several forward chemical screens have been used to identify compounds with relevance to autophagy. These cell-based phenotypic screens employed libraries of FDA-approved drugs and known bioactive compounds to identify chemical modulators of autophagy [114-117]. Various readouts were used to measure autophagic activity, including GFP-LC3 puncta formation, the clearance of mutant huntingtin and A53T $\alpha$ (alpha)-synuclein aggregates, and degradation of luciferase-fused LC3. Autophagy-related small molecules discovered in these screens can be broadly classified into two groups: (i) mTOR-dependent molecules, which consist of compounds that induce autophagy, and (ii) mTOR-independent molecules, which can be either inducers or suppressors of autophagy. The first group includes inhibitors of class I PI3K/Akt/mTOR signaling, such as allosteric and ATP-competitive mTOR kinase, Akt, PI3K, and dual mTOR/PI3K inhibitors. The second (mTORindependent) group includes inhibitors of cAMP-Epac-PLC- $\varepsilon$ (epsilon)-IP3 and Ca2+-calpain-Gs $\alpha$ (alpha) signaling [118].

Results from such chemical screens have been carried forward into cell and animal models of autophagy-related diseases. For example, small molecule enhancers 
of rapamycin [119] compounds were originally identified in a screen for enhancers of rapamycin-induced growth defects in S. cerevisiae [116]. Small-molecule enhancers (SMERS) 10, 18, or 28 were found to be active in mammalian cell models of Huntington's and Parkinson's disease, promoting autophagic clearance of mutant huntingtin and A53T $\alpha$ (alpha)-synuclein aggregates [116]. In vivo, SMERS protect against neurodegeneration in a Drosophila model of Huntington's disease [116].

Like the SMERs, the pan-nitric oxide synthase (NOS) inhibitor N $\omega$ (omega)nitro-L-arginine methyl ester hydrochloride (L-NAME) can block neurodegeneration in the same Drosophila Huntington's disease model and, similar to rapamycin, can clear mutant huntingtin aggregates in a zebrafish Huntington's disease model, suggesting that NOS inhibitors have therapeutic potential [120]. L-NAME triggers functional autophagy in a variety of cell types as measured by LC3 processing, the accumulation of RFP-LC3 puncta, and the ATG5-dependent clearance of mutant huntingtin aggregates [120]. While NOS inhibitors such as L-NAME decrease $\mathrm{NO}$, complementary experiments to increase $\mathrm{NO}$ levels using $\mathrm{NO}$ donors and $\mathrm{NO}$ synthase overexpression revealed an inhibitory role for NO in autophagy [120]. The MOA of NO was found to involve two different mechanisms of inhibiting autophagy. First, S-nitrosylation of JNK1 impairs phosphorylation of BCL2, leading to increased BCL2-belin 1 interaction. Second, S-nitrosylation of IKK $\beta$ promotes the TSC2-dependent activation of mTORC1 [120].

The role of HMGB1 in regulating mitophagy following rotenone-induced disruption of oxidative phosphorylation is a further example that highlights the utility of small molecule-gene interactions in identifying key autophagy pathways [51]. Mitochondrial stress mediated by rotenone, a small molecule that inhibits complex I in the electron transport chain, led to mitophagy that was facilitated by HMGB1 and its transcriptional target HSPB1. HMGB1 and HSPB1 were found to control mitochondrial homeostasis, as knockdown of either HMGB1 or HSPB1 resulted in perturbed morphology, glycolysis, ATP production, and mitochondrial fragmentation [51, 121]. Furthermore, rotenone-induced mitophagy was disrupted by cytochalasin D, suggesting a role for the actin cytoskeleton in HMGB1-HSPB1-mediated mitophagy [51].

To enhance the therapeutic utility of small molecules, the relationships between compound structure and biological activity (structure-activity relationships, or SARs) can be studied to determine which chemical groups present in the compound are important for activity and how modifications of the structure can improve selectivity and potency. In one example, Chen and colleagues synthesized a novel set of diphenylbutylpiperidines that demonstrated tenfold improved potency over lead compounds fluspirilene and penfluridol as measured by LC3-GFP puncta [122].

In addition to autophagy activators, autophagy inhibitors can also be useful as probes in determining whether a given state is dependent on classical autophagy or whether a compound truly induces autophagic flux. PI3K inhibitors 3-MA and wortmannin and the vacuolar $\mathrm{H}^{+}$ATPase inhibitor bafilomycin A1 are typically used for these purposes. Autophagy inhibitors can also have therapeutic potential. 
Chou and colleagues identified $\mathrm{DBeQ}$, a reversible ATP-competitive inhibitor of the p97 ATPase [123]. Inhibition of p97 following DBeQ treatments triggers diverse phenotypes, including a blockage of autophagosome maturation, activation of caspase 3 and 7, and inhibition of cancer cell proliferation, suggesting a potential therapeutic utility for p97 inhibitors in cancer [123].

Unlike the PI3K class I inhibitors discussed above, the discovery of potent and selective inhibitors of class III VPS34 has proven difficult. Class III VPS34 contains a smaller, more rigid ATP binding pocket compared to class I p110 $\gamma$, possibly adding to the difficulty in finding inhibitors [124]. 3-MA, a widely used inhibitor of VPS34, is typically used at millimolar concentrations, thus likely triggering many off-target effects that complicate results. To gain insight into developing VPS34 selective inhibitors, the structures of VPS34 in complexes with 3-MA, PIK-90, PIK-93, and PI103 were solved [124]. By employing structurebased design, Miller and colleagues were able to synthesize PT210, an analog of PIK-93, with ten-fold more selectivity for VPS34 than for class I p110 $\gamma$ [124]. Improved VPS34 inhibitors will be useful to probe the role of VPS34 in autophagy with more selectivity and without confounding effects of class I p110 $\gamma$ inhibition.

The success of small molecule screens in identifying and dissecting autophagy regulatory pathways is notable for its repercussions for therapeutics. The identification of new druggable targets may lead, after extensive optimization, to potential therapeutics in human diseases with autophagy-dependent and/or autophagymodulatory features. Experimental results such as those obtained using SMERs and NOS inhibitors suggest that small molecules may also be employed to target antibacterial autophagy at multiple stages.

\section{Concluding Remarks}

Recent research has provided significant insight into autophagic functions in immunity, including xenophagy, mitophagy, antigen presentation, vesicular trafficking and secretion, and cytokine activity. However, substantial questions remain regarding the mechanistic basis of such functions, as well as how individual disease risk alleles modulate these functions. In particular, studies of the microbiome may be critically important in understanding the relationship between xenophagy, immunity, and IBD. We anticipate that microbiome-wide studies (MWAS), in combination with detailed insights into the function of autophagy in health and disease, will help in the development of novel biological and chemical entities for the treatment of patients suffering from these inflammatory disorders.

Acknowledgments This work was supported by funding from the Crohn's and Colitis Foundation of America, and NIH grants DK043351, DK083756 and DK086502 to R.J.X. 


\section{References}

1. Mizushima N, Yoshimori T, Ohsumi Y (2011) The role of Atg proteins in autophagosome formation. Annu Rev Cell Dev Biol 27:107-132. Epub 2011/08/02

2. Li W, Zou W, Yang Y, Chai Y, Chen B, Cheng S et al (2012) Autophagy genes function sequentially to promote apoptotic cell corpse degradation in the engulfing cell. J Cell Biol 197(1):27-35. Epub 2012/03/28

3. Qu X, Zou Z, Sun Q, Luby-Phelps K, Cheng P, Hogan RN et al (2007) Autophagy genedependent clearance of apoptotic cells during embryonic development. Cell 128(5):931-946. Epub 2007/03/14

4. Pierdominici M, Vomero M, Barbati C, Colasanti T, Maselli A, Vacirca D et al (2012) Role of autophagy in immunity and autoimmunity, with a special focus on systemic lupus erythematosus. FASEB J 26(4):1400-1412. Epub 2012/01/17

5. Levine B, Mizushima N, Virgin HW (2011) Autophagy in immunity and inflammation. Nature 469(7330):323-335. Epub 2011/01/21

6. Kuballa P, Nolte WM, Castoreno AB, Xavier RJ (2012) Autophagy and the immune system. Annu Rev Immunol 30:611-646. Epub 2012/03/28

7. Deretic V (2011) Autophagy in immunity and cell-autonomous defense against intracellular microbes. Immunol Rev 240(1):92-104. Epub 2011/02/26

8. Franke A, McGovern DP, Barrett JC, Wang K, Radford-Smith GL, Ahmad T et al (2010) Genome-wide meta-analysis increases to 71 the number of confirmed Crohn's disease susceptibility loci. Nat Genet 42(12):1118-1125. Epub 2010/11/26

9. Barrett JC, Hansoul S, Nicolae DL, Cho JH, Duerr RH, Rioux JD et al (2008) Genome-wide association defines more than 30 distinct susceptibility loci for Crohn's disease. Nat Genet 40(8):955-962. Epub 2008/07/01

10. Rioux JD, Xavier RJ, Taylor KD, Silverberg MS, Goyette P, Huett A et al (2007) Genomewide association study identifies new susceptibility loci for Crohn disease and implicates autophagy in disease pathogenesis. Nat Genet 39(5):596-604. Epub 2007/04/17

11. Hampe J, Franke A, Rosenstiel P, Till A, Teuber M, Huse K et al (2007) A genome-wide association scan of nonsynonymous SNPs identifies a susceptibility variant for Crohn disease in ATG16L1. Nat Genet 39(2):207-211. Epub 2007/01/04

12. Anderson CA, Boucher G, Lees CW, Franke A, D'Amato M, Taylor KD et al (2011) Metaanalysis identifies 29 additional ulcerative colitis risk loci, increasing the number of confirmed associations to 47. Nat Genet 43(3):246-252. Epub 2011/02/08

13. Jostins L, Ripke S, Weersma RK, Duerr RH, McGovern DP, Hui KY et al (2012) Hostmicrobe interactions have shaped the genetic architecture of inflammatory bowel disease. Nature 491(7422):119-124. Epub 2012/11/07

14. Henckaerts L, Cleynen I, Brinar M, John JM, Van Steen K, Rutgeerts P et al (2011) Genetic variation in the autophagy gene ULK1 and risk of Crohn's disease. Inflamm Bowel Dis 17(6):1392-1397. Epub 2011/05/12

15. Brinar M, Vermeire S, Cleynen I, Lemmens B, Sagaert X, Henckaerts L et al (2012) Genetic variants in autophagy-related genes and granuloma formation in a cohort of surgically treated Crohn's disease patients. J Crohns Colitis 6(1):43-50. Epub 2012/01/21

16. Correia SC, Santos RX, Perry G, Zhu X, Moreira PI, Smith MA (2012) Mitochondrial importance in Alzheimer's, Huntington's and Parkinson's diseases. Adv Exp Med Biol 724:205221. Epub 2012/03/14

17. Youle RJ, Narendra DP (2011) Mechanisms of mitophagy. Nat Rev Mol Cell Biol 12(1):914. Epub 2010/12/24

18. Saitsu H, Nishimura T, Muramatsu K, Kodera H, Kumada S, Sugai K et al (2013.. Epub 2013/02/26) De novo mutations in the autophagy gene WDR45 cause static encephalopathy of childhood with neurodegeneration in adulthood. Nat Genet 45:445

19. Cullup T, Kho AL, Dionisi-Vici C, Brandmeier B, Smith F, Urry Z et al (2013) Recessive mutations in EPG5 cause Vici syndrome, a multisystem disorder with defective autophagy. Nat Genet 45(1):83-87. Epub 2012/12/12 
20. Inoue J, Nishiumi S, Fujishima Y, Masuda A, Shiomi H, Yamamoto K et al (2012) Autophagy in the intestinal epithelium regulates Citrobacter rodentium infection. Arch Biochem Biophys 521(1-2):95-101. Epub 2012/04/06

21. Saitoh T, Fujita N, Jang MH, Uematsu S, Yang BG, Satoh T et al (2008) Loss of the autophagy protein Atg16L1 enhances endotoxin-induced IL-1beta production. Nature 456(7219):264268. Epub 2008/10/14

22. Travassos LH, Carneiro LA, Ramjeet M, Hussey S, Kim YG, Magalhaes JG et al (2010) Nod1 and Nod2 direct autophagy by recruiting ATG16L1 to the plasma membrane at the site of bacterial entry. Nat Immunol 11(1):55-62. Epub 2009/11/10

23. Cooney R, Baker J, Brain O, Danis B, Pichulik T, Allan P et al (2010) NOD2 stimulation induces autophagy in dendritic cells influencing bacterial handling and antigen presentation. Nat Med 16(1):90-97. Epub 2009/12/08

24. Tooze SA, Yoshimori T (2010) The origin of the autophagosomal membrane. Nat Cell Biol 12(9):831-835. Epub 2010/09/03

25. Yang Z, Klionsky DJ (2010) Eaten alive: a history of macroautophagy. Nat Cell Biol 12(9):814-822. Epub 2010/09/03

26. Tsukada M, Ohsumi Y (1993) Isolation and characterization of autophagy-defective mutants of Saccharomyces cerevisiae. FEBS Lett 333(1-2):169-174. Epub 1993/10/25

27. Jung CH, Jun CB, Ro SH, Kim YM, Otto NM, Cao J et al (2009) ULK-Atg13-FIP200 complexes mediate mTOR signaling to the autophagy machinery. Mol Biol Cell 20(7):19922003. Epub 2009/02/20

28. Kamada Y, Yoshino K, Kondo C, Kawamata T, Oshiro N, Yonezawa K et al (2010) Tor directly controls the Atg1 kinase complex to regulate autophagy. Mol Cell Biol 30(4):10491058. Epub 2009/12/10

29. Cheong H, Nair U, Geng J, Klionsky DJ (2008) The Atg1 kinase complex is involved in the regulation of protein recruitment to initiate sequestering vesicle formation for nonspecific autophagy in Saccharomyces cerevisiae. Mol Biol Cell 19(2):668-681. Epub 2007/12/14

30. Chan EY, Longatti A, McKnight NC, Tooze SA (2009) Kinase-inactivated ULK proteins inhibit autophagy via their conserved C-terminal domains using an Atg13-independent mechanism. Mol Cell Biol 29(1):157-171. Epub 2008/10/22

31. Kageyama S, Omori H, Saitoh T, Sone T, Guan JL, Akira S et al (2011.. Epub 2011/04/29) The LC3 recruitment mechanism is separate from Atg9L1-dependent membrane formation in the autophagic response against Salmonella. Mol Biol Cell 22:2290

32. Itakura E, Kishi C, Inoue K, Mizushima N (2008) Beclin 1 forms two distinct phosphatidylinositol 3-kinase complexes with mammalian Atg14 and UVRAG. Mol Biol Cell 19(12):5360-5372. Epub 2008/10/10

33. Matsunaga K, Saitoh T, Tabata K, Omori H, Satoh T, Kurotori N et al (2009) Two Beclin 1-binding proteins, Atg14L and Rubicon, reciprocally regulate autophagy at different stages. Nat Cell Biol 11(4):385-396. Epub 2009/03/10

34. Zhong Y, Wang QJ, Li X, Yan Y, Backer JM, Chait BT et al (2009) Distinct regulation of autophagic activity by Atg14L and Rubicon associated with Beclin 1-phosphatidylinositol-3kinase complex. Nat Cell Biol 11(4):468-476. Epub 2009/03/10

35. Sun Q, Fan W, Chen K, Ding X, Chen S, Zhong Q (2008) Identification of Barkor as a mammalian autophagy-specific factor for Beclin 1 and class III phosphatidylinositol 3-kinase. Proc Natl Acad Sci U S A 105(49):19211-19216. Epub 2008/12/04

36. Matsunaga K, Morita E, Saitoh T, Akira S, Ktistakis NT, Izumi T et al (2010) Autophagy requires endoplasmic reticulum targeting of the PI3-kinase complex via Atg14L. J Cell Biol 190(4):511-521. Epub 2010/08/18

37. Takahashi Y, Coppola D, Matsushita N, Cualing HD, Sun M, Sato Y et al (2007) Bif-1 interacts with Beclin 1 through UVRAG and regulates autophagy and tumorigenesis. Nat Cell Biol 9(10):1142-1151. Epub 2007/09/25

38. Liang C, Feng P, Ku B, Dotan I, Canaani D, Oh BH et al (2006) Autophagic and tumour suppressor activity of a novel Beclin1-binding protein UVRAG. Nat Cell Biol 8(7):688-699. Epub 2006/06/27 
39. Liang C, Lee JS, Inn KS, Gack MU, Li Q, Roberts EA et al (2008) Beclin1-binding UVRAG targets the class $\mathrm{C}$ Vps complex to coordinate autophagosome maturation and endocytic trafficking. Nat Cell Biol 10(7):776-787. Epub 2008/06/17

40. Mizushima N, Noda T, Yoshimori T, Tanaka Y, Ishii T, George MD et al (1998) A protein conjugation system essential for autophagy. Nature 395(6700):395-398. Epub 1998/10/06

41. Mizushima N, Sugita H, Yoshimori T, Ohsumi Y (1998) A new protein conjugation system in human. The counterpart of the yeast Apg 12p conjugation system essential for autophagy. J Biol Chem 273(51):33889-33892. Epub 1998/12/16

42. Fujita N, Saitoh T, Kageyama S, Akira S, Noda T, Yoshimori T (2009) Differential involvement of Atg16L1 in Crohn disease and canonical autophagy: analysis of the organization of the Atg16L1 complex in fibroblasts. J Biol Chem 284(47):32602-32609. Epub 2009/09/29

43. Noda NN, Ohsumi Y, Inagaki F (2010) Atg8-family interacting motif crucial for selective autophagy. FEBS Lett 584(7):1379-1385. Epub 2010/01/20

44. Kabeya Y, Mizushima N, Ueno T, Yamamoto A, Kirisako T, Noda T et al (2000) LC3, a mammalian homologue of yeast Apg8p, is localized in autophagosome membranes after processing. EMBO J 19(21):5720-5728. Epub 2000/11/04

45. Kabeya Y, Mizushima N, Yamamoto A, Oshitani-Okamoto S, Ohsumi Y, Yoshimori T (2004) LC3, GABARAP and GATE16 localize to autophagosomal membrane depending on form-II formation. J Cell Sci 117(Pt 13):2805-2812. Epub 2004/06/01

46. Satoo K, Noda NN, Kumeta H, Fujioka Y, Mizushima N, Ohsumi Y et al (2009) The structure of Atg4B-LC3 complex reveals the mechanism of LC3 processing and delipidation during autophagy. EMBO J 28(9):1341-1350. Epub 2009/03/27

47. Weidberg H, Shvets E, Shpilka T, Shimron F, Shinder V, Elazar Z (2010) LC3 and GATE-16/ GABARAP subfamilies are both essential yet act differently in autophagosome biogenesis. EMBO J 29(11):1792-1802. Epub 2010/04/27

48. Kimura S, Noda T, Yoshimori T (2008) Dynein-dependent movement of autophagosomes mediates efficient encounters with lysosomes. Cell Struct Funct 33(1):109-122. Epub 2008/04/05

49. Korolchuk VI, Saiki S, Lichtenberg M, Siddiqi FH, Roberts EA, Imarisio S et al (2011) Lysosomal positioning coordinates cellular nutrient responses. Nat Cell Biol 13(4):453-460. Epub 2011/03/12

50. Lavoie JN, Hickey E, Weber LA, Landry J (1993) Modulation of actin microfilament dynamics and fluid phase pinocytosis by phosphorylation of heat shock protein 27. J Biol Chem 268(32):24210-24214. Epub 1993/11/15

51. Tang D, Kang R, Livesey KM, Kroemer G, Billiar TR, Van Houten B et al (2011) Highmobility group box 1 is essential for mitochondrial quality control. Cell Metab 13(6):701711. Epub 2011/06/07

52. Settembre C, Di Malta C, Polito VA, Garcia Arencibia M, Vetrini F, Erdin S et al (2011) TFEB links autophagy to lysosomal biogenesis. Science 332(6036):1429-1433. Epub $2011 / 05 / 28$

53. Yu XJ, McGourty K, Liu M, Unsworth KE, Holden DW (2010) pH sensing by intracellular Salmonella induces effector translocation. Science 328(5981):1040-1043. Epub 2010/04/17

54. Patel JC, Hueffer K, Lam TT, Galan JE (2009) Diversification of a Salmonella virulence protein function by ubiquitin-dependent differential localization. Cell 137(2):283-294. Epub 2009/04/22

55. Bakowski MA, Braun V, Lam GY, Yeung T, Heo WD, Meyer T et al (2010) The phosphoinositide phosphatase SopB manipulates membrane surface charge and trafficking of the Salmonella-containing vacuole. Cell Host Microbe 7(6):453-462. Epub 2010/06/15

56. Zheng YT, Shahnazari S, Brech A, Lamark T, Johansen T, Brumell JH (2009) The adaptor protein $\mathrm{p} 62 / \mathrm{SQSTM} 1$ targets invading bacteria to the autophagy pathway. J Immunol 183(9):5909-5916. Epub 2009/10/09

57. Thurston TL, Ryzhakov G, Bloor S, von Muhlinen N, Randow F (2009) The TBK1 adaptor and autophagy receptor NDP52 restricts the proliferation of ubiquitin-coated bacteria. Nat Immunol 10(11):1215-1221. Epub 2009/10/13 
58. Korioth F, Gieffers C, Maul GG, Frey J (1995) Molecular characterization of NDP52, a novel protein of the nuclear domain 10, which is redistributed upon virus infection and interferon treatment. J Cell Biol 130(1):1-13. Epub 1995/07/01

59. Sternsdorf T, Jensen K, Zuchner D, Will H (1997) Cellular localization, expression, and structure of the nuclear dot protein 52. J Cell Biol 138(2):435-448. Epub 1997/07/28

60. Cemma M, Kim PK, Brumell JH (2011) The ubiquitin-binding adaptor proteins p62/ SQSTM1 and NDP52 are recruited independently to bacteria-associated microdomains to target Salmonella to the autophagy pathway. Autophagy 7(3):341-345. Epub 2010/11/17

61. Mostowy S, Sancho-Shimizu V, Hamon M, Simeone R, Brosch R, Johansen T et al (2011. Epub 2011/06/08) p62 and NDP52 target Intracytosolic Shigella and Listeria to different autophagy pathways. J Biol Chem 286:26987

62. Veiga E, Cossart P (2005) Ubiquitination of intracellular bacteria: a new bacteria-sensing system? Trends Cell Biol 15(1):2-5. Epub 2005/01/18

63. Birmingham CL, Smith AC, Bakowski MA, Yoshimori T, Brumell JH (2006) Autophagy controls Salmonella infection in response to damage to the Salmonella-containing vacuole. J Biol Chem 281(16):11374-11383. Epub 2006/02/24

64. Huett A, Heath RJ, Begun J, Sassi SO, Baxt LA, Vyas JM et al (2012) The LRR and RING domain protein LRSAM1 is an E3 ligase crucial for ubiquitin-dependent autophagy of intracellular Salmonella Typhimurium. Cell Host Microbe 12(6):778-790. Epub 2012/12/19

65. Reis BS, Mucida D (2012) The role of the intestinal context in the generation of tolerance and inflammation. Clin Dev Immunol 2012:157948. Epub 2011/09/29

66. Lapaquette P, Glasser AL, Huett A, Xavier RJ, Darfeuille-Michaud A (2010) Crohn's diseaseassociated adherent-invasive E. coli are selectively favoured by impaired autophagy to replicate intracellularly. Cell Microbiol 12(1):99-113. Epub 2009/09/15

67. Kuballa P, Huett A, Rioux JD, Daly MJ, Xavier RJ (2008) Impaired autophagy of an intracellular pathogen induced by a Crohn's disease associated ATG16L1 variant. PLoS One 3(10): 3391 . Epub 2008/10/15

68. McCarroll SA, Huett A, Kuballa P, Chilewski SD, Landry A, Goyette P et al (2008) Deletion polymorphism upstream of IRGM associated with altered IRGM expression and Crohn's disease. Nat Genet 40(9):1107-1112. Epub 2009/01/24

69. Brest P, Lapaquette P, Souidi M, Lebrigand K, Cesaro A, Vouret-Craviari V et al (2011) A synonymous variant in IRGM alters a binding site for miR-196 and causes deregulation of IRGM-dependent xenophagy in Crohn's disease. Nat Genet 43(3):242-245. Epub 2011/02/01

70. Tong Y, Giaime E, Yamaguchi H, Ichimura T, Liu Y, Si H et al (2012) Loss of leucine-rich repeat kinase 2 causes age-dependent bi-phasic alterations of the autophagy pathway. Mol Neurodegener 7:2. Epub 2012/01/11

71. Plowey ED, Cherra SJ 3rd, Liu YJ, Chu CT (2008) Role of autophagy in G2019S-LRRK2associated neurite shortening in differentiated SH-SY5Y cells. J Neurochem 105(3):10481056. Epub 2008/01/10

72. Gardet A, Benita Y, Li C, Sands BE, Ballester I, Stevens C et al (2010) LRRK2 is involved in the IFN-gamma response and host response to pathogens. J Immunol 185(9):5577-5585. Epub 2010/10/06

73. Kumar D, Nath L, Kamal MA, Varshney A, Jain A, Singh S et al (2010) Genome-wide analysis of the host intracellular network that regulates survival of Mycobacterium tuberculosis. Cell 140(5):731-743. Epub 2010/03/10

74. Intemann CD, Thye T, Niemann S, Browne EN, Amanua Chinbuah M, Enimil A et al (2009) Autophagy gene variant IRGM -261T contributes to protection from tuberculosis caused by Mycobacterium tuberculosis but not by M. africanum strains. PLoS Pathog 5(9):e1000577. Epub 2009/09/15

75. Chiodini RJ, Chamberlin WM, Sarosiek J, McCallum RW (2012) Crohn's disease and the mycobacterioses: a quarter century later. Causation or simple association? Crit Rev Microbiol 38(1):52-93. Epub 2012/01/17 
76. Orvedahl A, Sumpter R Jr, Xiao G, Ng A, Zou Z, Tang Y et al (2011) Image-based genomewide siRNA screen identifies selective autophagy factors. Nature 480(7375):113-117. Epub $2011 / 10 / 25$

77. Matsuda N, Sato S, Shiba K, Okatsu K, Saisho K, Gautier CA et al (2010) PINK1 stabilized by mitochondrial depolarization recruits Parkin to damaged mitochondria and activates latent Parkin for mitophagy. J Cell Biol 189(2):211-221. Epub 2010/04/21

78. Narendra D, Kane LA, Hauser DN, Fearnley IM, Youle RJ (2010) p62/SQSTM1 is required for Parkin-induced mitochondrial clustering but not mitophagy; VDAC1 is dispensable for both. Autophagy 6(8):1090-1106. Epub 2010/10/05

79. Jin SM, Lazarou M, Wang C, Kane LA, Narendra DP, Youle RJ (2010) Mitochondrial membrane potential regulates PINK1 import and proteolytic destabilization by PARL. J Cell Biol 191(5):933-942. Epub 2010/12/01

80. Geisler S, Holmstrom KM, Skujat D, Fiesel FC, Rothfuss OC, Kahle PJ et al (2010) PINK1/ Parkin-mediated mitophagy is dependent on VDAC1 and p62/SQSTM1. Nat Cell Biol 12(2):119-131. Epub 2010/01/26

81. Tanaka A, Cleland MM, Xu S, Narendra DP, Suen DF, Karbowski M et al (2010) Proteasome and p97 mediate mitophagy and degradation of mitofusins induced by Parkin. J Cell Biol 191(7):1367-1380. Epub 2010/12/22

82. Gegg ME, Cooper JM, Chau KY, Rojo M, Schapira AH, Taanman JW (2010) Mitofusin 1 and mitofusin 2 are ubiquitinated in a PINK1/parkin-dependent manner upon induction of mitophagy. Hum Mol Genet 19(24):4861-4870. Epub 2010/09/28

83. Ziviani E, Tao RN, Whitworth AJ (2010) Drosophila parkin requires PINK1 for mitochondrial translocation and ubiquitinates mitofusin. Proc Natl Acad Sci U S A 107(11):50185023. Epub 2010/03/03

84. Fimia GM, Stoykova A, Romagnoli A, Giunta L, Di Bartolomeo S, Nardacci R et al (2007) Ambra1 regulates autophagy and development of the nervous system. Nature 447(7148):1121-1125. Epub 2007/06/26

85. Van Humbeeck C, Cornelissen T, Hofkens H, Mandemakers W, Gevaert K, De Strooper B et al (2011) Parkin interacts with ambral to induce mitophagy. J Neurosci 31(28):1024910261. Epub 2011/07/15

86. Dengjel J, Schoor O, Fischer R, Reich M, Kraus M, Muller M et al (2005) Autophagy promotes MHC class II presentation of peptides from intracellular source proteins. Proc Natl Acad Sci U S A 102(22):7922-7927. Epub 2005/05/17

87. Kasai M, Tanida I, Ueno T, Kominami E, Seki S, Ikeda T et al (2009) Autophagic compartments gain access to the MHC class II compartments in thymic epithelium. J Immunol 183(11):7278-7285. Epub 2009/11/17

88. Schmid D, Pypaert M, Munz C (2007) Antigen-loading compartments for major histocompatibility complex class II molecules continuously receive input from autophagosomes. Immunity 26(1):79-92. Epub 2006/12/22

89. Lee HK, Mattei LM, Steinberg BE, Alberts P, Lee YH, Chervonsky A et al (2010) In vivo requirement for Atg5 in antigen presentation by dendritic cells. Immunity 32(2):227-239. Epub 2010/02/23

90. Ireland JM, Unanue ER (2011) Autophagy in antigen-presenting cells results in presentation of citrullinated peptides to CD4 T cells. J Exp Med 208(13):2625-2632. Epub 2011/12/14

91. Paludan C, Schmid D, Landthaler M, Vockerodt M, Kube D, Tuschl T et al (2005) Endogenous MHC class II processing of a viral nuclear antigen after autophagy. Science 307(5709):593596. Epub 2004/12/14

92. Wildenberg ME, Vos AC, Wolfkamp SC, Duijvestein M, Verhaar AP, Te Velde AA et al (2012) Autophagy attenuates the adaptive immune response by destabilizing the immunologic synapse. Gastroenterology 142(7):1493-503 e6. Epub 2012/03/01

93. Bevins CL, Salzman NH (2011) Paneth cells, antimicrobial peptides and maintenance of intestinal homeostasis. Nat Rev Microbiol 9(5):356-368. Epub 2011/03/23

94. Cadwell K, Patel KK, Maloney NS, Liu TC, Ng AC, Storer CE et al (2010) Virus-plussusceptibility gene interaction determines Crohn's disease gene Atg16L1 phenotypes in intestine. Cell 141(7):1135-1145. Epub 2010/07/07 
95. Cadwell K, Liu JY, Brown SL, Miyoshi H, Loh J, Lennerz JK et al (2008) A key role for autophagy and the autophagy gene Atg1611 in mouse and human intestinal Paneth cells. Nature 456(7219):259-U62

96. Cadwell K, Patel KK, Komatsu M, Virgin HW, Stappenbeck TS (2009) A common role for Atg16L1, Atg5 and Atg7 in small intestinal Paneth cells and Crohn disease. Autophagy $5(2): 250-252$

97. Biswas A, Liu YJ, Hao L, Mizoguchi A, Salzman NH, Bevins CL et al (2010) Induction and rescue of Nod2-dependent Th1-driven granulomatous inflammation of the ileum. Proc Natl Acad Sci U S A 107(33):14739-14744. Epub 2010/08/04

98. Kaser A, Lee AH, Franke A, Glickman JN, Zeissig S, Tilg H et al (2008) XBP1 links ER stress to intestinal inflammation and confers genetic risk for human inflammatory bowel disease. Cell 134(5):743-756. Epub 2008/09/09

99. Yilmaz OH, Katajisto P, Lamming DW, Gultekin Y, Bauer-Rowe KE, Sengupta S et al (2012) mTORC1 in the Paneth cell niche couples intestinal stem-cell function to calorie intake. Nature 486(7404):490-495. Epub 2012/06/23

100. Dideberg V, Kristjansdottir G, Milani L, Libioulle C, Sigurdsson S, Louis E et al (2007) An insertion-deletion polymorphism in the interferon regulatory factor 5 (IRF5) gene confers risk of inflammatory bowel diseases. Hum Mol Genet 16(24):3008-3016. Epub 2007/09/21

101. Zhou R, Yazdi AS, Menu P, Tschopp J (2011) A role for mitochondria in NLRP3 inflammasome activation. Nature 469(7329):221-225. Epub 2010/12/03

102. Bulua AC, Simon A, Maddipati R, Pelletier M, Park H, Kim KY et al (2011) Mitochondrial reactive oxygen species promote production of proinflammatory cytokines and are elevated in TNFR1-associated periodic syndrome (TRAPS). J Exp Med 208(3):519-533. Epub 2011/02/02

103. Wen H, Gris D, Lei Y, Jha S, Zhang L, Huang MT et al (2011) Fatty acid-induced NLRP3ASC inflammasome activation interferes with insulin signaling. Nat Immunol 12(5):408415. Epub 2011/04/12

104. Plantinga TS, Crisan TO, Oosting M, van de Veerdonk FL, de Jong DJ, Philpott DJ et al (2011) Crohn's disease-associated ATG16L1 polymorphism modulates pro-inflammatory cytokine responses selectively upon activation of NOD2. Gut 60(9):1229-1235. Epub 2011/03/17

105. Lee J, Kim HR, Quinley C, Kim J, Gonzalez-Navajas J, Xavier R et al (2012) Autophagy suppresses interleukin-1beta (IL-1beta) signaling by activation of $\mathrm{p} 62$ degradation via lysosomal and proteasomal pathways. J Biol Chem 287(6):4033-4040. Epub 2011/12/15

106. Raju D, Hussey S, Ang M, Terebiznik MR, Sibony M, Galindo-Mata E et al (2012) Vacuolating cytotoxin and variants in Atg16L1 that disrupt autophagy promote Helicobacter pylori infection in humans. Gastroenterology 142(5):1160-1171. Epub 2012/02/16

107. Singh SB, Ornatowski W, Vergne I, Naylor J, Delgado M, Roberts E et al (2010) Human IRGM regulates autophagy and cell-autonomous immunity functions through mitochondria. Nat Cell Biol 12(12):1154-1165. Epub 2010/11/26

108. Parkes M, Barrett JC, Prescott NJ, Tremelling M, Anderson CA, Fisher SA et al (2007) Sequence variants in the autophagy gene IRGM and multiple other replicating loci contribute to Crohn's disease susceptibility. Nat Genet 39(7):830-832. Epub 2007/06/08

109. Behrends C, Sowa ME, Gygi SP, Harper JW (2010) Network organization of the human autophagy system. Nature 466(7302):68-76. Epub 2010/06/22

110. Rozenknop A, Rogov VV, Rogova NY, Lohr F, Guntert P, Dikic I et al (2011) Characterization of the interaction of GABARAPL-1 with the LIR motif of NBR1. J Mol Biol 410(3):477487. Epub 2011/05/31

111. Kraft C, Peter M, Hofmann K (2010) Selective autophagy: ubiquitin-mediated recognition and beyond. Nat Cell Biol 12(9):836-841. Epub 2010/09/03

112. Ong SE, Schenone M, Margolin AA, Li X, Do K, Doud MK et al (2009) Identifying the proteins to which small-molecule probes and drugs bind in cells. Proc Natl Acad Sci U S A 106(12):4617-4622. Epub 2009/03/04

113. Lamb J, Crawford ED, Peck D, Modell JW, Blat IC, Wrobel MJ et al (2006) The connectivity map: using gene-expression signatures to connect small molecules, genes, and disease. Science 313(5795):1929-1935. Epub 2006/09/30 
114. Zhang L, Yu J, Pan H, Hu P, Hao Y, Cai W et al (2007) Small molecule regulators of autophagy identified by an image-based high-throughput screen. Proc Natl Acad Sci U S A 104(48):19023-19028. Epub 2007/11/21

115. Balgi AD, Fonseca BD, Donohue E, Tsang TC, Lajoie P, Proud CG et al (2009) Screen for chemical modulators of autophagy reveals novel therapeutic inhibitors of mTORC1 signaling. PLoS One 4(9):e7124. Epub 2009/09/23

116. Sarkar S, Perlstein EO, Imarisio S, Pineau S, Cordenier A, Maglathlin RL et al (2007) Small molecules enhance autophagy and reduce toxicity in Huntington's disease models. Nat Chem Biol 3(6):331-338. Epub 2007/05/09

117. Farkas T, Hoyer-Hansen M, Jaattela M (2009) Identification of novel autophagy regulators by a luciferase-based assay for the kinetics of autophagic flux. Autophagy 5(7):1018-1025. Epub 2009/08/05

118. Fleming A, Noda T, Yoshimori T, Rubinsztein DC (2011) Chemical modulators of autophagy as biological probes and potential therapeutics. Nat Chem Biol 7(1):9-17. Epub $2010 / 12 / 18$

119. Semerari A, Cucchi M, Dimaggio G, Cavadini D, Carcione A, Battelli V et al (2012.. Epub 2012/08/22) The development of the Metacognition Assessment Interview: instrument description, factor structure and reliability in a non-clinical sample. Psychiatry Res 200:890

120. Sarkar S, Korolchuk VI, Renna M, Imarisio S, Fleming A, Williams A et al (2011) Complex inhibitory effects of nitric oxide on autophagy. Mol Cell 43(1):19-32. Epub 2011/07/06

121. Kang R, Livesey KM, Zeh HJ 3rd, Loze MT, Tang D (2011) Metabolic regulation by HMGB1mediated autophagy and mitophagy. Autophagy 7(10):1256-1258. Epub 2011/06/22

122. Chen G, Xia H, Cai Y, Ma D, Yuan J, Yuan C (2011) Synthesis and SAR study of diphenylbutylpiperidines as cell autophagy inducers. Bioorg Med Chem Lett 21(1):234-239. Epub 2010/12/04

123. Chou TF, Brown SJ, Minond D, Nordin BE, Li K, Jones AC et al (2011) Reversible inhibitor of p97, DBeQ, impairs both ubiquitin-dependent and autophagic protein clearance pathways. Proc Natl Acad Sci U S A 108(12):4834-4839. Epub 2011/03/09

124. Miller S, Tavshanjian B, Oleksy A, Perisic O, Houseman BT, Shokat KM et al (2010) Shaping development of autophagy inhibitors with the structure of the lipid kinase Vps34. Science 327(5973):1638-1642. Epub 2010/03/27 